\title{
Quantum-Hall quantum bits
}

\author{
S.-R. Eric Yang, ${ }^{1,2}$ John Schliemann, ${ }^{1,3}$ and A. H. MacDonald ${ }^{1}$ \\ ${ }^{1}$ Department of Physics, University of Texas, Austin, TX 78712 \\ ${ }^{2}$ Department of Physics, Korea University, Seoul, Korea \\ ${ }^{3}$ Department of Physics and Astronomy, University of Basel, Switzerland
}

(Received 27 June 2002; published 2 October 2002)

\begin{abstract}
Bilayer quantum-Hall systems can form collective states in which electrons exhibit spontaneous interlayer phase coherence. We discuss the possibility of using bilayer quantum dot many-electron states with this property to create two-level systems that have potential advantages as quantum bits.
\end{abstract}

DOI: 10.1103/PhysRevB.66.153302

PACS number(s): 03.67.Lx, 73.21.La, 73.43.Lp

Over the past several years there has been a great deal of interest in solid-state two-level systems that could serve as quantum computing bits (qubits), ${ }^{1}$ and thereby enable largescale quantum computation. In order for a two-level system to be useful as a quantum bit, it must be possible to maintain coherent quantum evolution over time scales long compared to those required for its intentional manipulation. This is particularly difficult to achieve in a solid-state environment because of the presence of many degrees of freedom, electronic, photonic, and nuclear, which interact relatively strongly. The spin degree of freedom of individual electrons ${ }^{2}$ or nuclei ${ }^{3}$ in a semiconductor, and the total charge degree of freedom of a small superconductor ${ }^{4}$ are among the possibilities that have been been advanced for crafting solid state qubits. In this paper we propose a different possibility, qubits based on the the total pseudospin of double-layer quantum dots in the quantum-Hall regime. We suggest an experiment that could be used to demonstrate their quantum coherence, and discuss some of their potential advantages.

The two-level system we discuss is related to the spontaneous-coherence states that occur in bulk bilayer quantum-Hall systems. ${ }^{5-8}$ These states have a gap for charged excitations, which is entirely due to electronelectron interactions. They can be viewed either as pseudospin ferromagnets or as a Bose condensate ${ }^{9}$ of pairs formed between electrons in one layer and holes in a Landau level of an adjoining layer. Their low-energy physics can be described ${ }^{10}$ by an effective model usually expressed in terms of a pseudospin quantum field. The pseudospin in these systems is the quantum which-layer degree of freedom. An electron that is in an eigenstate of the $\hat{z}$-component of the pseudospin operator (with eigenvalue $\pm 1 / 2$ ) is definitely in one of the layers, while one with quantum uncertainty in its layer index is in a state with a pseudospin component in the $\hat{x}-\hat{y}$ plane. The qubits we propose are, however, based on manyelectron eigenstates in small double-layer quantum dot ${ }^{11}$ systems, like those realized recently by Tarucha and co-workers $^{12}$ and illustrated schematically in Fig. 1. They have cylindrical symmetry about an axis along which a strong magnetic field $B \hat{z}$ can be applied. The dot area is controlled by a side gate, which provides an approximately harmonic lateral confining potential that stabilizes finite area quasi-two-dimensional quantum dots.
If we assume that the quantum dots of interest are sufficiently small to suppress spatial variation of the pseudospin, the effective-field theory ${ }^{10}$ has a single pseudospin collective coordinate $\left(T_{x}, T_{y}, T_{z}\right)$ and an effective Hamiltonian:

$$
\mathcal{H}=\frac{8 \pi l^{2} \beta}{N} T_{z}^{2}-\Delta_{v} T_{z}-\alpha \Delta_{t} T_{x} .
$$

In Eq. (1), $T_{z}=-N / 2,-N / 2+1, \ldots, N / 2$ is half the difference between electron numbers in right and left dots, $N$ is the total number of electrons in the double dot, $l$ is the magnetic length defined by $2 \pi l^{2} B=h c / e=\Phi_{0}$, where $\Phi_{0}$ is the magnetic-flux quantum, $\Delta_{v}$ is an adjustable interlayer bias voltage, $\Delta_{t}$ is the splitting of single-particle energy levels in the double-layer system due to interlayer tunneling, $\alpha<1$

a)

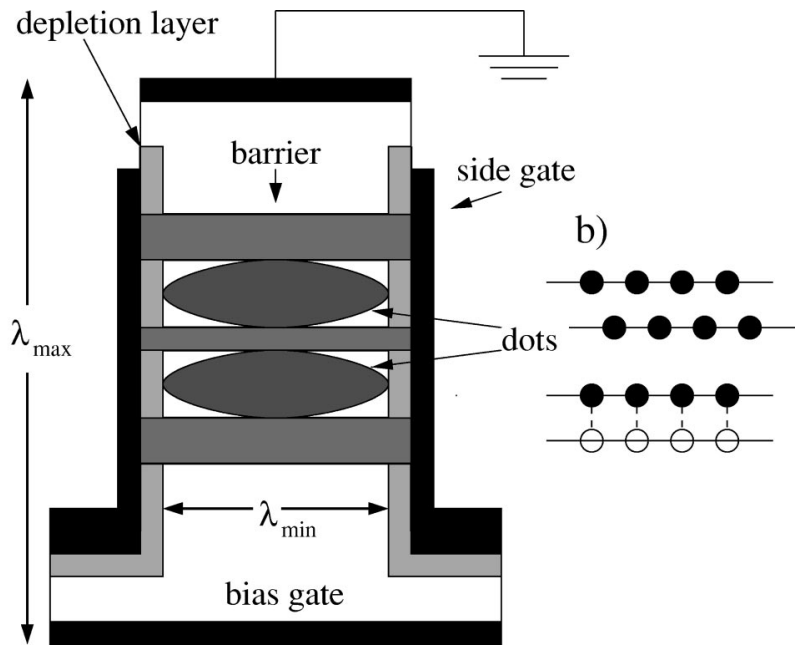

FIG. 1. Schematic illustration of a pillar bilayer double-dot system. We consider the situation in which a strong magnetic field is applied along the axis of the pillar. The barriers that confine electrons to the two GaAs layers in the pillar are established by the epitaxial growth of $(\mathrm{Al}, \mathrm{Ga}) \mathrm{As}$ layers. The maximum phonon wavelengths in the pillar $\left(\lambda_{\max }\right)$ are the geometrical metrics associated with the structure's fundamental vibration modes, represented in this figure by the pillar height. The minimum wavelengths $\lambda_{\min }$ for phonons that couple effectively to collective electronic states of the dot are comparable to the dot radius or the interlayer distance. Since $\lambda_{\text {max }}$ is comparable to $\lambda_{\text {min }}$, only the fundamental and at most of few harmonics will be important in decohering these collective state qubits. 


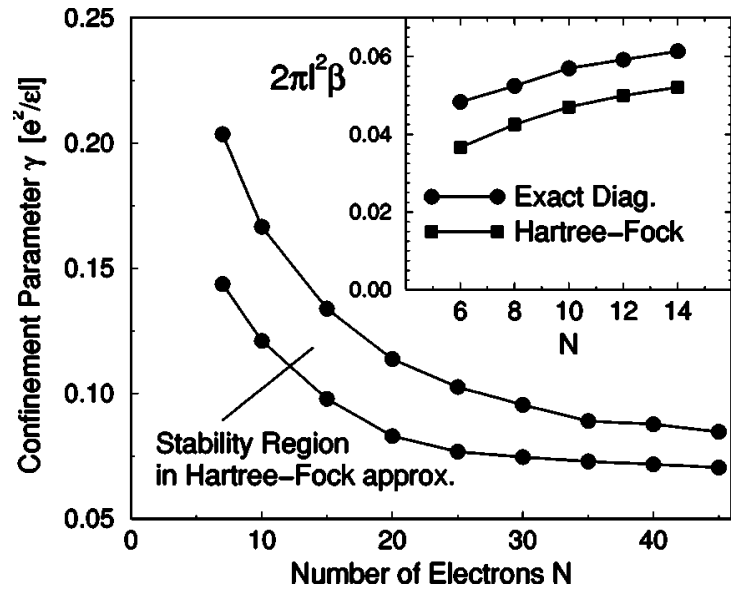

FIG. 2. Hartree-Fock stability range of the bilayer coherent state as a function of electron number $N$. The inset displays values of $2 \pi l^{2} \beta$ obtained by fitting the effective Hamiltonian to exactdiagonalization and Hartree-Fock theory results. The unit of $2 \pi l^{2} \beta$ is $e^{2} / \epsilon l$.

accounts for microscopic quantum fluctuations in the manyelectron state, and $\beta$ is discussed below. For small $\Delta_{t}$ and the ratio $\Delta_{v} N / 8 \pi l^{2} \beta$ equal to an appropriate integer, the spectrum of this Hamiltonian has two low-lying levels separated from higher-lying states by a large gap. This two-level system is our proposed qubit.

The mean-field theory of bilayer quantum-Hall ferromagnets, like Ref. 9, the Bardeen-Cooper-Schrieffer (BCS) theory of superconductivity, provides both valuable insight into the nature of these states and a practical tool for quantitative estimates. For bilayer quantum dot systems, ${ }^{13,14}$ the mean-field state consists of a Slater determinant of bilayer symmetric, for example, single-particle orbitals with angular momenta $m=0, \ldots, N-1$ that establish coherence between the layers even when $\Delta_{t}=0$. The bilayer coherent state is stable when the occupied symmetric single-particle orbitals have mean-field eigenenergies that are (i) lower than those of symmetric states with larger angular momenta and (ii) lower than those of any antisymmetric state. ${ }^{14}$ Note that these quasiparticle energies are split by a gap, analogous to the BCS gap of a superconductor, that can be entirely due to interactions and does not vanish for $\Delta_{t} \rightarrow 0$. For weak confinement or strong fields, ${ }^{14}$ electron-electron interactions dominate and favor a fractional average occupation of angular-momentum states, violating the first condition. For strong confinement or weak fields, ${ }^{14}$ average occupation numbers larger than 1 are favored, violating the second condition. As illustrated in Fig. 2 , the double-dot coherent state is stable only over a finite range of confinement strengths, $\gamma=m \Omega^{2} l^{2}$, which narrows with increasing particle number $N$ ( $\Omega$ is the frequency of the harmonic potential). The value of $\beta$ can be estimated, using either mean field or exact diagonalization calculations, from the dependence of the ground-state pseudospin polarization on bias potential. From the inset of Fig. 2, we see that $2 \pi l^{2} \beta=0.06 e^{2} / \epsilon l$ at $N=14$, compared to the bulk value $2 \pi l^{2} \beta=0.09 e^{2} / \epsilon l .{ }^{10}$ The results of Fig. 2 were obtained with the interlayer separation equal to $l$ and with $\Delta_{t}$ and $\Delta_{v}$ set to zero.

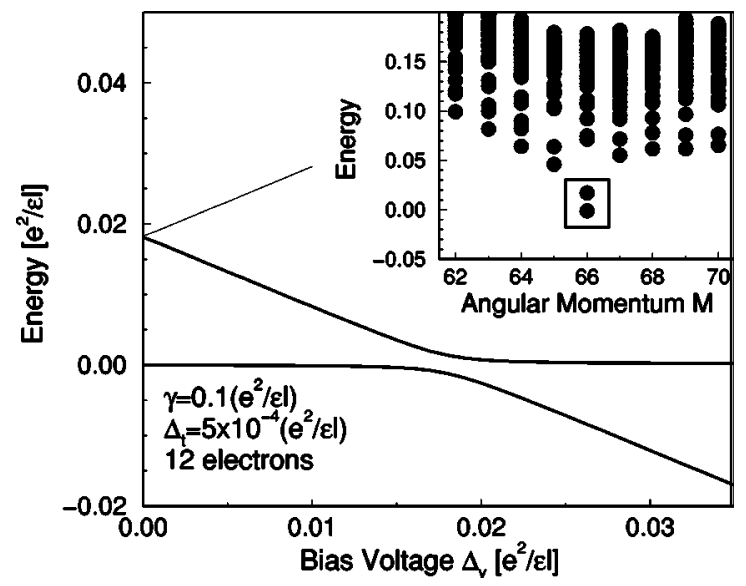

FIG. 3. Dependence of the three lowest eigenvalues with total angular momentum $M=M_{G S}$ on bias voltage $\Delta_{v}$. The inset shows the low-energy part of the many-electron energy spectrum [in units of $\left.e^{2} / \epsilon l\right]$ as a function of total angular momentum $M$ for 12 electrons at $\Delta_{v}=0$ and at the strength of the confinement potential $\gamma$ $=0.1 e^{2} / \epsilon l . M_{G S}=66$ for $N=12$. This figure demonstrates that the two-level system of our proposed qubit is cleanly separated from other many-electron states.

In the inset of Fig. 3, we plot the exact spectrum of the many-particle Hamiltonian for 12 electrons in a double-layer quantum dot system as a function of total angular momentum $M$. The collective state discussed above has quasiparticles with angular momentum $m=0, \ldots, N-1$, and so occurs at total angular momentum $M_{D L C S}=N(N-1) / 2$. Exact diagonalization calculations show that the double-layer coherent state is the ground state over a narrow range of field strengths. The results plotted in Fig. 3 are for $\gamma=0.1 e^{2} / \epsilon l$, which is in the middle of the stability range for $N=12$. Comparing with Fig. 2, we see that Hartree-Fock theory only slightly overestimates the confinement strength at which the double-layer coherent state occurs. The main part of Fig. 3 shows the dependence of the three lowest eigenvalues on $\Delta_{v}$ for $\Delta_{t}$ equal to a small positive value. The first anticrossing takes place near $\Delta_{v}=0.02 e^{2} / \epsilon l$, consistent with the effective model prediction, $\Delta_{v}=8 \pi l^{2} \beta / N$. These results demonstrate that the effective Hamiltonian accurately describes the lowenergy part of the dependence of the microscopic Hamiltonian's spectrum on $\Delta_{t}$ and $\Delta_{v}$, which will figure importantly in our discussion. The lowest-energy states that appear at $M>M_{G S}$ in Fig. 3 are edge excitations of the dot, which form a part of our proposed qubit's environment as discussed below.

For $N$ even, assumed in the following, we choose the many-body states $|0\rangle$ and $|1\rangle\left(T_{z}\right.$ eigenvalues are 0 and 1$)$ as the orthogonal states of the proposed qubit. (For $N$ odd, the optimal orthogonal states would be $|1 / 2\rangle$ and $|-1 / 2\rangle$.) At zero bias voltage, the ground state is nondegenerate and, for small $\Delta_{t}$, has nearly definite $T_{z}$. The truncated two-level Hamiltonian is given up to a constant by

$$
H_{P}=\left(\frac{4 \pi l^{2} \beta}{N}-\frac{\Delta_{v}}{2}\right) \sigma_{z}-\frac{\Delta}{2} \sigma_{x}
$$


where $\Delta=\alpha \Delta_{t} \sqrt{(N+2) N} / 2$, and $\sigma_{z}$ and $\sigma_{x}$ are Pauli spin matrices in the space of the qubit. The tunneling term in the Hamiltonian, which is very small for the quantum-dot systems we have in mind, is effective only near the resonance situation, $\Delta_{v}=8 \pi l^{2} \beta / N$, where it splits the degeneracy that would otherwise occur. From Fig. 3 we find that $\Delta=2.57$ $\times 10^{-3} e^{2} / \epsilon l$, corresponding to $\alpha=0.86$.

In this paragraph, we propose an experiment that we believe is feasible, and which can be used to study the coherent quantum evolution of this qubit. If the bias voltage is held at zero, where the $\hat{z}$ component of the qubit effective field dominates, for a sufficiently long time the qubit will reach its ground state $|0\rangle$. When the bias voltage is tuned to resonance, the qubit evolves under the influence of the $\hat{x}$-direction Zeeman field. If after a time $T$, the bias voltage returns to zero, the qubit will have a finite probability amplitude for being in the $|1\rangle$ state, i.e., for having transferred an electron between layers of quantum dot. We require that equilibrium should be reestablished by charge flowing through the external circuit used to control the bias voltage, a property that requires the dot contact resistances to be smaller than the off-resonance intradot resistance. If this procedure is repeated many times with a repetition period $T_{\text {repeat }}$ that is longer than the equilibration time, the current flowing between layers through the external circuit measures the probability for transferring an electron,

$$
I=e\left(\frac{1}{2}-\frac{1-2 p}{2} \cos (\Delta T / 2 \hbar)\right) / T_{\text {repeat }} .
$$

Here $p \neq 0$ is small if the resonance condition is established rapidly compared to $\hbar / \Delta$ as we discuss below. A measurement of oscillating current as a function of time-onresonance $T$ would establish quantum coherence for this potential qubit.

There are, of course, no genuine two-level systems in nature, and the one under discussion here is not an exception. Even discounting coupling to phonons, nuclear spins, and other degrees of freedom present in the solid-state environment, the electron Hamiltonian has a large number of eigenstates. A practical requirement ${ }^{15}$ for an effective two-level system is that its operational time scale $\hbar / \Delta$ should be long compared to the scale which breaks adiabaticity between the two-level system and its electronic environment. If the pseudospin effective Hamiltonian fully describes the double layer quantum dot, this time scale would be $\hbar /\left(2 \pi l^{2} \beta\right)$. If rotational symmetry of the Hamiltonian is broken, on the other hand, edge states are the lowest-lying electronic excitations outside of the two-level system. As illustrated in Fig. 3, the size of these energies for realistic double-layer quantum dots is $\sim 1.0 \mathrm{meV}$ for $e^{2} / \epsilon l \sim 10 \mathrm{meV}$. The tunneling amplitude between bilayer systems can be varied over a very wide range because of its exponential dependence on $\mathrm{Al}$ content in the barrier separating the quantum wells and on the width of this barrier. For $\Delta_{t}=0.0003 \mathrm{meV}$ and $N=40$, the gap $\Delta$ is $0.001 \mathrm{meV}$, safely smaller than edge excitation energies, and the operational time of the quantum bit is $\sim 10^{-9} \mathrm{~s}$, making it possible to adjust the bias potential sufficiently rapidly to achieve $p \ll 1$ in Eq. (3).
Bilayer quantum-Hall systems are similar ${ }^{7}$ in many respects to the Josephson coupled superconductors (but also show differences, ${ }^{8}$ ) and there are both similarities and differences between the experiment we propose above and the coherent single-Cooper-pair box effect realized ${ }^{16}$ by Nakamura et al. In the Nakamura experiment, the qubit is formed by states that differ by 1 in the number of Cooper pairs on one side of the junction, whereas in the excitonic insulator language our qubit is formed by states that differ by 1 in the number of electron-hole pairs in the system. Since a new electron-hole pair is formed by moving a single electron from one layer to the other, the current expression in our case is smaller by a factor of 2 . The two qubits share the property that, because of the collective behavior of many electrons, coherence can be established even though the systems are composed of a relatively large number of electrons. The phase coherence time of bulk bilayer quantum-Hall systems has been estimated ${ }^{6}$ to be $\tau_{\phi} \sim 2 \times 10^{-10} \mathrm{~s}$, most likely ${ }^{8}$ due to coupling between collective degrees of freedom and electronic excitations near the boundaries between the incompressible and compressible regions that occur in all bulk samples. This coherence time is shorter than the period we propose above for a typical Rabi-oscillating current made from quantum-Hall quantum bits. However, as explained above, this decoherence mechanism is not operative for quantum dot systems. Instead, the dominant decoherence mechanism is likely to be piezoelectric coupling to longwavelength phonons, nabbed ${ }^{17}$ as the likely culprit for single-electron double dots. ${ }^{17,18}$ For collective qubits, like the ones we propose, only phonons with wavelengths comparable to the entire electronic system can effectively decohere. We illustrate this point in Fig. 4 by plotting the dependence of the change in $\Delta_{v}$ on $N$ in the presence of a potential

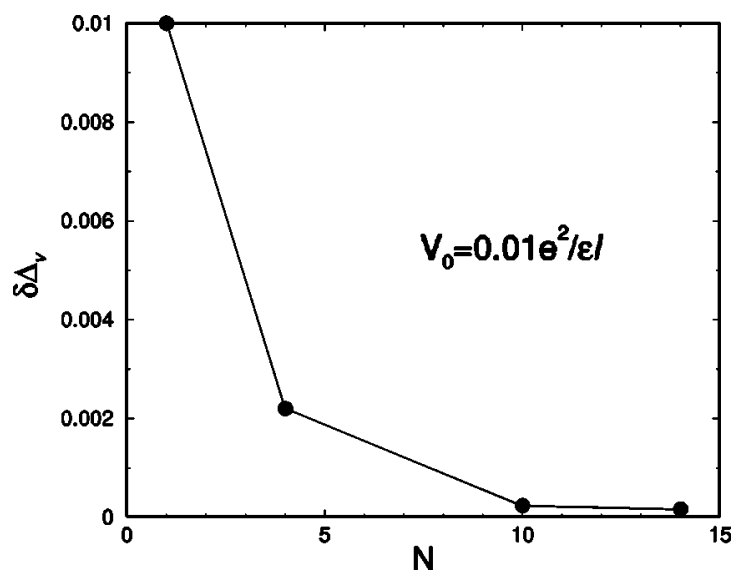

FIG. 4. Dependence of the change in the qubit parameter $\Delta_{v}$ on $N$ in the presence of a fluctuation in the potential difference between the two layers that is localized at the center of the quantum dot. This quantity has been evaluated by performing self-consistent Hartree-Fock calculations at $\Delta_{t}=0$ and evaluating the energy difference between states that differ by 1 in the number of electrons in the top layer. The potential fluctuation strength has been chosen so that it alters $\Delta_{v}$ by $0.01 e^{2} / \epsilon l$ for a single electron. Electronic correlations in large- $N$ collective states suppress the system's response to the fluctuation potential, and reduces the change in $\Delta_{v}$, which results from the same potential fluctuation. 
fluctuation located at the center of the quantum dot. For large $N$, correlations between electrons in the collective state suppress response to the localized potential fluctuation, and limit its effectiveness in decohering the qubit. We expect that phonons will be an especially weak decoherence mechanism for these qubits because the maximum phonon wavelength, established by size quantization, is only slightly larger than the minimum effective phonon wavelength, established by the size of the double-dot system. The fact that the decoherence time is already $\sim 10^{-10} \mathrm{~s}$ in the bulk, suggests that very long coherence times will be achievable.

Solid-state quantum computing clearly presents even more daunting obstacles than merely coherent time evolution of solid-state qubits. The problems that would need to be solved to control the entanglement of these quantum-Hall qubits are similar to entanglement strategies for the case of of Cooper pair box qubits. ${ }^{4}$ We believe that experimental study of the novel qubit proposed here, which occurs in a physical system that has already been achieved, ${ }^{12}$ could make valuable contribution to the growing understanding of decoherence in solids.

We are grateful for valuable conversations with G. Austing, S. Tarucha, D. Loss, Y. Nakamura, and P. Zoller. S.R.E.Y was supported by the KOSEF Quantum-functional Semiconductor Research Center at Dongguk University and by the Korea Research Foundation under Grant No. 1998015-D00114. J.S. was supported by the Deutsche Forschungsgemeinschaft. Work at the University of Texas was supported by the Welch Foundation and the by the National Science Foundation under Grant No. DMR0115947.
${ }^{1}$ For a review see, for example, M.A. Nielsen and I.L. Chuang, Quantum Computation and Quantum Information (Cambridge University Press, Cambridge, England, 2000).

${ }^{2}$ D. Loss and D.P. DiVincenzo, Phys. Rev. A 57, 120 (1998).

${ }^{3}$ B.E. Kane, Nature (London) 393, 133 (1998).

${ }^{4}$ Y. Makhlin et al., Nature (London) 398, 305 (1999).

${ }^{5}$ S.Q. Murphy et al., Phys. Rev. Lett. 72, 728 (1994); I.B. Spielman et al., ibid. 84, 5808 (2000); 87, 36803 (2001); L. Balents and L. Radzihovsky, ibid. 86, 1825 (2001); A. Stern et al., ibid. 86, 1829 (2001); J. Schliemann et al., ibid. 86, 1849 (2001); E. Demler et al., ibid. 86, 1852 (2001); K. Yang, ibid. 87, 056802 (2001).

${ }^{6}$ For reviews see J.P. Eisenstein, Phys. World 14(6), 30 (2001); B. G. Levi, Phys. Today 54(5), 14 (2001); S.M. Girvin, e-print cond-mat/0108181.

${ }^{7}$ X.G. Wen and A. Zee, Phys. Rev. Lett. 69, 1811 (1992); Z.F. Ezawa and A. Iwazaki, Int. J. Mod. Phys. B 19, 3205 (1992); M. Fogler and F. Wilczek, Phys. Rev. Lett. 86, 1833 (2001).
${ }^{8}$ Y. Joglekar and A.H. MacDonald, Phys. Rev. Lett. 87, 196802 (2001).

${ }^{9}$ A.H. MacDonald, Physica B 298, 129 (2001).

${ }^{10}$ K. Moon et al., Phys. Rev. A A51, 5138 (1995).

${ }^{11}$ For recent reviews see L.P. Kouwenhoven et al., Rep. Prog. Phys. 64, 701 (2001); L.P. Kouwenhoven and P.L. McEuen, in Nanoscience and Technology, edited by G. Timp (Springer-Verlag, New York, 1999).

${ }^{12}$ D.G. Austing et al., Physica B 249-251, 206 (2000); Y. Tokura et al., Physica E (Amsterdam) 6, 676 (2000).

${ }^{13}$ S.-R. Eric Yang et al., Phys. Rev. Lett. 71, 3194 (1993); A.H. MacDonald et al., Aust. J. Phys. 46, 345 (1993).

${ }^{14}$ J. Hu et al., Phys. Rev. B 54, 8616 (1996).

${ }^{15}$ J. Schliemann et al., Phys. Rev. B 63, 085311 (2001).

${ }^{16}$ Y. Nakamura et al., Nature (London) 398, 786 (1999).

${ }^{17}$ T. Fujisawa et al., Science 282, 932 (1998).

${ }^{18}$ M. Bayer et al., Science 291, 451 (2001); R.H. Blick et al., Phys. Rev. Lett. 80, 4032 (1998); T. Brandes et al., Phys. Rev. B 64, 035319 (2001); A.W. Holleitner et al., Phys. Rev. Lett. 87, 256802 (2001) 